ISSN = 1980-993X-doi:10.4136/1980-993X
www.agro.unitau.br/ambi-agua
E-mail: ambi-agua@agro.unitau.br
Tel.: (12) 3625-4116

\title{
Calibração de sondas TDR em condições de laboratório
}

(doi:10.4136/ambi-agua.43)

\author{
Dione Inês Christ Milani' ${ }^{1}$ Maria Hermínia Ferreira Tavares²; Clementina \\ Scherpinski ${ }^{3}$ \\ ${ }^{1}$ Faculdades Assis Gurgaz - Mestre em Engenharia Agrícola \\ E-mail: dioneicmilani@hotmail.com \\ ${ }^{2}$ Universidade Estadual do Oeste do Paraná - Docente do Programa de Pós-Graduação em Engenharia \\ Agrícola \\ E-mail: mhstavar@certto.com.br \\ ${ }^{3}$ Universidade Estadual do Oeste do Paraná - Mestre em Engenharia Agrícola \\ E-mail: scherpinski@unioeste.br
}

\section{RESUMO}

Dentre os métodos indiretos para a determinação da umidade volumétrica do solo $\left(\theta_{\mathrm{v}}\right)$, a técnica da Reflectometria no Domínio do Tempo (TDR) tem recebido crescente atenção. Como toda técnica, apresenta vantagens e desvantagens, entre elas a maior desvantagem é a necessidade de calibração. No estudo aqui apresentado, foram obtidos valores da constante dielétrica aparente do solo $\left(\mathrm{K}_{\mathrm{a}}\right)$ e umidade volumétrica $\left(\theta_{\mathrm{v}}\right)$ para um Latossolo Vermelho, por meio de um experimento de laboratório e utilizadas dezessete sondas de TDR, sendo duas sondas originais e quinze confeccionadas pela própria equipe. $O$ trabalho objetivou: a) calibrar as dezessete sondas, em condições de laboratório, comparando-se seis modelos de regressão para cada sonda; b) verificar a viabilidade do emprego das sondas manufaturadas. Constatou-se que o melhor ajuste de regressão para todas as sondas foi a equação polinomial cúbica e que as sondas montadas pela equipe apresentaram desempenho semelhante ao das sondas originais.

Palavras-chave: constante dielétrica aparente; calibração; Reflectometria no Domínio do Tempo; umidade volumétrica do solo.

\section{TDR probes calibration in laboratory conditions}

\section{ABSTRACT}

Among the indirect methods to evaluate the volumetric soil water content $\left(\theta_{\mathrm{v}}\right)$, the Time Domain Reflectometry (TDR) technique is receiving growing attention. As any technique, it presents advantages and disadvantages: the most serious disadvantage is the calibration necessity. At the present study, values of apparent dielectric constant $\left(\mathrm{K}_{\mathrm{a}}\right)$ and volumetric water content $\left(\theta_{\mathrm{v}}\right)$ for a Red Latosol were obtained in a laboratory experiment, using seventeen probes of TDR, from which two were original and fifteen were manufactured by the staff. This study aimed: a) to calibrate the seventeen probes, in laboratory conditions, comparing six regression models for each probe; b) to verify the viability to use the manufactured probes. It was found out that the best regression model was the cubic polynomial model and that the manufactured probes presented performances comparable to the original ones.

Keywords: apparent dielectric constant; calibration; Time Domain Reflectometry; volumetric soil water content. 


\section{INTRODUÇÃO}

Existem vários métodos indiretos para a determinação do conteúdo da água do solo, dentre eles a TDR (Reflectometria no Domínio do Tempo), pela qual o tempo de trânsito de uma onda eletromagnética por uma haste colocada no solo fornece a constante dielétrica aparente do solo $\left(K_{a}\right)$, dada pela Equação 1:

$$
K_{a}=\left(\frac{t \times c}{2 L}\right)^{2}
$$

na qual $t$ é o tempo de deslocamento (em s) da onda eletromagnética, $c$ é a velocidade da luz $\left(3.0 \times 10^{8} \mathrm{~m} / \mathrm{s}\right)$ e $L$ é o comprimento da haste da sonda $(\mathrm{em} \mathrm{m})$.

Existem vantagens e desvantagens no uso da técnica da TDR: para Tommaselli e Bacchi (2001), a vantagem é a rapidez na obtenção da umidade e a desvantagem é a dependência do tipo de solo (mineral ou orgânico), materiais magnéticos e a calibração trabalhosa. Os fatos das sondas TDR poderem ser acopladas a multiplexadores (Coelho et al., 2006) ou a outros tipos de sensores, tais como penetrômetros (Manieri et al., 2007), constituem excepcional vantagem da técnica da TDR. Segundo Noborio (2001), a TDR proporciona a repetibilidade das leituras, a não destruição da região amostrada, a não emissão de radiações ionizantes, portabilidade e facilidade de acoplamento a dispositivos coletores de dados, tendo como desvantagem o custo elevado.

Para diminuir os custos, sondas nacionais têm sido desenvolvidas. Villwock et al. (2004) construíram e avaliaram sondas, em condições de campo e laboratório, mostrando a boa qualidade das sondas montadas pela equipe local, a um custo nove vezes menor do que o custo das sondas originais importadas. Outra iniciativa para a diminuição de custos foi o desenvolvimento de aplicativos nacionais para a aquisição e gerenciamento dos dados (Tavares et al., 2006), uma vez que os programas importados apresentam alto custo.

Para Topp et al. (1980), a relação empírica entre a constante dielétrica aparente $K_{a}$ e o conteúdo volumétrico da água $\theta_{v}$ é independente do tipo do solo, de sua densidade, da temperatura e do conteúdo de sais solúveis. Esses autores consideram que, para freqüências entre 1 e $20 \mathrm{GHz}$, a única variável que afeta a resposta dielétrica do solo é o conteúdo da água no solo, com um peso relativo de cerca de $90 \%$. Assim, a constante dielétrica aparente $\mathrm{K}_{\mathrm{a}}$ pode ser usada para determinar $\theta_{v}$.

As pesquisas existentes demonstram que há constante procura de um modelo universal de calibração para os equipamentos TDR, devendo-se, entretanto, levar em consideração a composição atípica de alguns solos. Segundo Roth et al. (1990), há variações na curva de calibração para solos que apresentam maior susceptibilidade aos campos magnéticos, como por exemplo, os solos com altos teores de óxidos de ferro. Segundo Tommaselli e Bacchi (2001), os trabalhos realizados a respeito do assunto levaram a três tipos de abordagem na formulação dos modelos das curvas de calibração: abordagens empíricas, semi-empíricas e físicas.

Em um trabalho pioneiro, Topp et al. (1980) estudaram a relação empírica entre $\mathrm{K}_{\mathrm{a}}$ e $\theta_{\mathrm{v}}$ (umidade volumétrica) para quatro solos minerais e encontraram como ajuste um único modelo linear cúbico. Tommaselli e Bacchi (2001) salientam que os modelos indicados pelos fabricantes dos equipamentos TDR devem ser abandonados, já que desconsideram as peculiaridades dos solos, devendo-se realizar novas calibrações. Esse autor encontra como melhor ajuste modelos cúbicos para cinco solos diferentes, tanto para os dados de cada um 
dos solos tratados isoladamente, assim como para o conjunto dos dados tratados de forma conjunta. Os modelos cúbicos de calibração foram obtidos também por outros autores (Tommaselli e Bacchi, 2001; Villwock et al., 2004).

Os objetivos deste trabalho foram: a) calibrar dezessete sondas de TDR, das quais duas eram originais e quinze fabricadas pela equipe; b) verificar a viabilidade do emprego das sondas manufaturadas. Os dados foram obtidos em condições de laboratório, em um Latossolo Vermelho com alto teor de argila e procurando-se a melhor equação de regressão, de forma a garantir maior precisão na obtenção da umidade volumétrica.

\section{MATERIAL E MÉTODOS}

O experimento foi realizado com um Latossolo Vermelho, coletado no Núcleo Experimental de Engenharia Agrícola da Universidade Estadual do Oeste do Paraná, na cidade de Cascavel, com coordenadas geográficas $24^{0} 58^{\prime}$ de latitude Sul e $26^{0}$ de longitude Oeste de Greenwich. A caracterização dos atributos físicos e químicos do solo estudado é apresentada na Tabela 1.

Tabela 1. Atributos físicos e químicos do Latossolo Vermelho empregado no experimento (camada de $5,0$ a $25,0 \mathrm{~cm})$.

\begin{tabular}{lrrr}
\hline Atributo & \multicolumn{1}{c}{ Valor } & Atributo & \multicolumn{1}{c}{ Valor } \\
\hline Areia & $3,0 \%$ & $\mathrm{pH}\left(\mathrm{CaCl}_{2}\right)$ & 5,80 \\
Silte & $27,9 \%$ & $\mathrm{C}$ & $23,22 \mathrm{mgdm}^{-3}$ \\
Argila & $69,1 \%$ & $\mathrm{P}$ & $5,70 \mathrm{mgdm}^{-3}$ \\
Densidade do solo & $1,31 \mathrm{Mgm}^{-3}$ & $\mathrm{Fe}$ & $68,10 \mathrm{mgdm}^{-3}$ \\
Densidade de partículas & $2,92 \mathrm{Mgm}^{-3}$ & $\mathrm{~K}^{+}$ & $0,14 \mathrm{cmoldm}^{-3}$ \\
Porosidade Total & $55,0 \%$ & $\mathrm{Ca}^{2+}$ & $7,73 \mathrm{cmoldm}^{-3}$ \\
Macroporosidade & $10,4 \%$ & $\mathrm{Mg}^{2+}$ & $4,60 \mathrm{cmoldm}^{-3}$ \\
Microporosidade & $44,6 \%$ & $\mathrm{H}^{+}+\mathrm{Al}^{3+}$ & $4,61 \mathrm{cmoldm}^{-3}$ \\
Umidade volumétrica na capacidade de & $0,405 \mathrm{~m}^{3} \mathrm{~m}^{-3}$ & & \\
campo & & & \\
Umidade volumétrica no ponto de & $0,253 \mathrm{~m}^{3} \mathrm{~m}^{-3}$ & & \\
murcha permanente & & & \\
\hline
\end{tabular}

Foram utilizadas sondas de três hastes, cujos comprimentos variaram de $17,0 \mathrm{~cm}$ a 42,0 $\mathrm{cm}$, fabricadas segundo Villwock et al. (2004) e acopladas a um equipamento TDR modelo TRASE 6050X1, da Soil Moisture Equipment Corp ${ }^{*}$, o qual foi empregado de acordo com as instruções do fabricante (Soil Moisture Equipment Corporation, 1998).

O solo foi coletado na camada de 5,0 a $25,0 \mathrm{~cm}$ de profundidade, seco ao ar por 144 horas e peneirado com peneira de $2 \mathrm{~mm}$, obtendo-se assim TFSA (terra fina seca ao ar). Depois de peneirado, o solo foi colocado em oito cilindros de PVC, com capacidade individual de 15 litros, sendo compactado de modo a manter sua densidade o mais uniforme possível. Após 24 horas, os cilindros foram pesados para a obtenção do valor da densidade de

*Referência ao fabricante não implica indicação por parte dos autores. 
solo úmido $\rho_{u}$, após o que as sondas TDR foram cuidadosamente introduzidas no solo de cada cilindro, procurando-se evitar a formação de lacunas de ar entre as hastes e o solo, o que poderia comprometer as leituras.

Após cada leitura com o equipamento TDR, foram retiradas quatro amostras deformadas de solo de cada cilindro, as quais foram pesadas e levadas à estufa por 24 horas para a obtenção da umidade gravimétrica $\mu$, a qual foi empregada no cálculo da umidade volumétrica $\theta_{v}$, conforme a Equação 2:

$$
\theta_{v}=\frac{\rho_{u}}{\frac{1}{\mu}+1}
$$

Em função do volume de solo colocado em cada cilindro $\left(0,015 \mathrm{~m}^{3}\right)$, foram utilizados $250 \mathrm{ml}$ de água em cada umedecimento. A cada operação de umedecimento, o solo era colocado em um recipiente cilíndrico maior, sendo a água pulverizada e misturada. Depois de umedecido e homogeneizado, o solo era novamente compactado nos cilindros, os quais eram hermeticamente fechados, evitando-se assim perda de água por evaporação. As leituras foram sempre realizadas em intervalos de 24 horas, obtendo-se nove níveis diferentes de umidade.

Foi realizada uma leitura por sonda para cada recipiente, totalizando quatro repetições para cada nível de umidade. Os números 1 a 15 referem-se às sondas fabricadas, enquanto os números 16 e 17 foram reservados para as sondas originais.

A Figura 1 mostra três exemplares de sondas utilizadas neste experimento, sendo a central uma sonda original e as laterais sondas manufaturadas pela equipe.

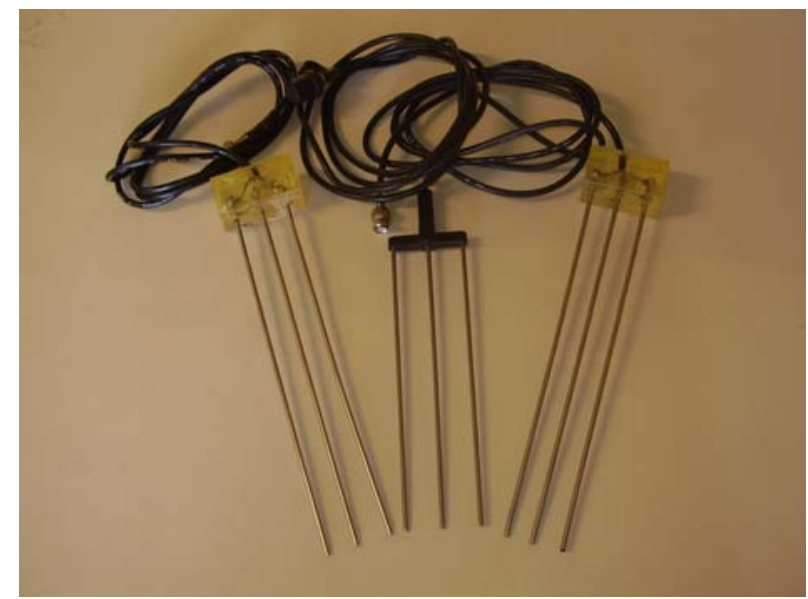

Figura 1. Exemplares de sondas TDR utilizadas no experimento.

O teste de Scott-Knott foi aplicado no nível de 5\% de significância, comparando-se os resultados das médias e separando-se as variáveis em grupos distintos.

\section{RESULTADOS E DISCUSSÃO}

O intervalo de umidade volumétrica obtido nas condições do trabalho foi de $22,0 \%$ a $36,0 \%$. Os comportamentos da constante dielétrica aparente do solo para as diferentes sondas podem ser comparados na Figura 2. Podemos observar que a sonda 2 apresentou a maior variabilidade e que todas as sondas mostraram comportamento semelhante, sempre com o 
valor médio de cada leitura superando a mediana e indicando uma variação maior com o aumento de $K_{a}$.

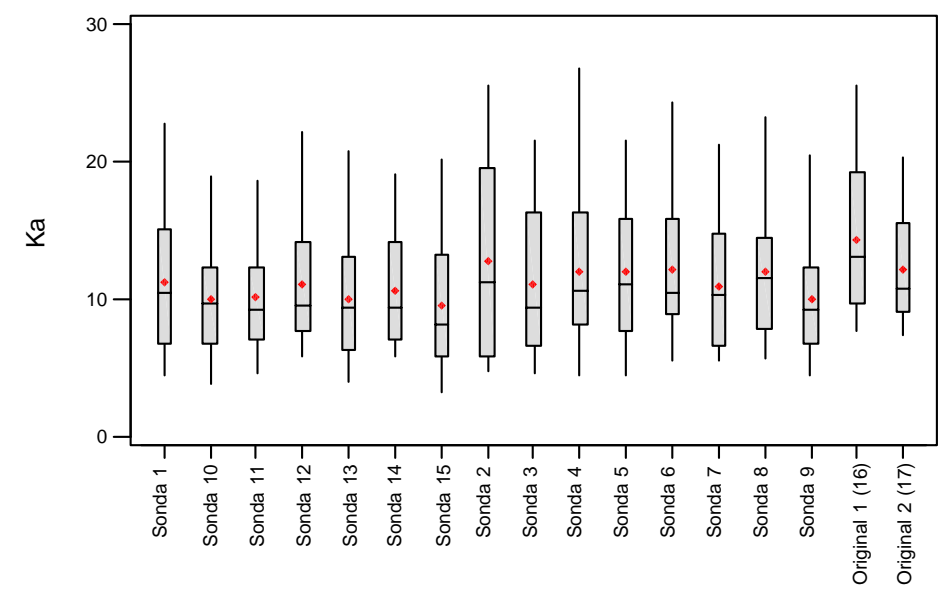

Figura 2. Variação de $K_{a}$ para as diferentes sondas. As médias estão indicadas em vermelho.

O comprimento de cada sonda, número de pontos ajustados, desvio padrão, resultado do teste de semelhança e o valor de $p$ da análise de variância da equação de regressão cúbica ajustada podem ser visualizados na Tabela 2, sendo a diferença entre o número de pontos ajustados decorrente da falta de leitura em determinadas ocasiões. No teste de semelhança, letras iguais significam sondas iguais em média. $\mathrm{O}$ valor de $\mathrm{p}$ mostrou-se significativo para todas as regressões.

Os resultados do desvio padrão foram relativamente grandes, porém, semelhantes, pois a variação de umidade entre cada medida, utilizando $250 \mathrm{ml}$ de água para cada intervalo, foi pré-estabelecida. O teste de Scott-Knott mostrou que as sondas 2, 4, 5, 6, 8, 16 e 17 foram iguais, em média, entre si, enquanto que as sondas $1,3,7,9,10,11,12,13,14$ e 15 formaram outro grupo de sondas semelhantes. A análise de variância mostrou que os dados tiveram regressão significativa.

Foram ajustados seis modelos de regressão para cada sonda, sendo eles: linear, quadrático, cúbico, logarítmico, potencial e exponencial. $\mathrm{O}$ modelo polinomial cúbico foi o que melhor se ajustou às regressões, sendo a única exceção a sonda 7 , para a qual o melhor modelo ajustado foi o potencial, apresentando, porém pequena diferença em relação ao modelo cúbico. As sondas 14 e 16 mostraram os melhores coeficientes $\mathrm{R}^{2}$, com valores 0,9415 e 0,9427 respectivamente. A sonda 7 obteve o menor coeficiente $\mathrm{R}^{2}$ mas, mesmo assim, seu resultado pode ser considerado ótimo. Os coeficientes de determinação $\mathrm{R}^{2}$ e $\overline{R^{2}}$ mostraram-se bons em todos os casos. A Tabela 3 mostra o melhor ajuste de regressão, bem como o coeficiente de determinação $R^{2}$ e o coeficiente de determinação ajustado $\overline{R^{2}}$, o qual leva em consideração o número de dados ajustados para cada sonda. 
MILANI, D. I. C.; TAVARES, M. H. F.; SCHERPINSKI, C. Calibração de sondas TDR em condições de laboratório. Ambi-Agua, Taubaté, v. 3, n. 1, p. 68-75, 2008. (doi:10.4136/ambi-agua.43)

Tabela 2. Dados relevantes para cada sonda.

\begin{tabular}{cccccc}
\hline Sonda & $\begin{array}{c}\text { Comprimento } \\
(\mathbf{c m})\end{array}$ & $\begin{array}{c}\mathbf{N}^{\mathbf{o}} \mathbf{\text { de pontos }} \\
\text { ajustados }\end{array}$ & Desvio Padrão & Teste de Scott-Knott & Valor de $\boldsymbol{p}$ \\
\hline 1 & 17,4 & 36 & 4,788 & $\mathrm{a}$ & 0,000 \\
2 & 17,3 & 24 & 6,977 & $\mathrm{~b}$ & 0,000 \\
3 & 17,4 & 32 & 4,935 & $\mathrm{a}$ & 0,000 \\
4 & 17,3 & 35 & 5,346 & $\mathrm{~b}$ & 0,000 \\
5 & 22,1 & 36 & 4,800 & $\mathrm{~b}$ & 0,000 \\
6 & 21,7 & 33 & 4,655 & $\mathrm{~b}$ & 0,000 \\
7 & 22,2 & 34 & 4,327 & $\mathrm{a}$ & 0,000 \\
8 & 22,1 & 26 & 5,267 & $\mathrm{~b}$ & 0,000 \\
9 & 32,0 & 35 & 4,170 & $\mathrm{a}$ & 0,000 \\
10 & 31,9 & 34 & 3,895 & $\mathrm{a}$ & 0,000 \\
11 & $31,9 \mathrm{~cm}$ & 34 & 3,743 & $\mathrm{a}$ & 0,000 \\
12 & $41,4 \mathrm{~cm}$ & 27 & 4,054 & $\mathrm{a}$ & 0,000 \\
13 & $41,4 \mathrm{~cm}$ & 34 & 4,271 & $\mathrm{a}$ & 0,000 \\
14 & $41,6 \mathrm{~cm}$ & 35 & 3,792 & $\mathrm{a}$ & 0,000 \\
15 & $41,6 \mathrm{~cm}$ & 35 & 4,365 & $\mathrm{a}$ & 0,000 \\
16 & $20,0 \mathrm{~cm}$ & 36 & 5,399 & $\mathrm{~b}$ & 0,000 \\
17 & $30,0 \mathrm{~cm}$ & 35 & 3,700 & $\mathrm{~b}$ & 0,000 \\
\hline
\end{tabular}

Tabela 3. Modelos ajustados e respectivos coeficientes de determinação para cada sonda.

\begin{tabular}{ccrc}
\hline Sonda & \multicolumn{1}{c}{ Modelo } & \multicolumn{1}{c}{$R^{2}$} & $\overline{R^{2}}$ \\
\hline 1 & $\theta=-4 \mathrm{E}-05 \mathrm{~K}_{\mathrm{a}}{ }^{3}+0.0014 \mathrm{~K}_{\mathrm{a}}{ }^{2}-0.0081 \mathrm{~K}_{\mathrm{a}}+0.2492$ & 0.83358 & 0.81798 \\
2 & $\theta=-3 \mathrm{E}-05 \mathrm{~K}_{\mathrm{a}}{ }^{3}+0.0012 \mathrm{~K}_{\mathrm{a}}{ }^{2}-0.0089 \mathrm{~K}_{\mathrm{a}}+0.2578$ & 0.86195 & 0.84125 \\
3 & $\theta=-3 \mathrm{E}-05 \mathrm{~K}_{\mathrm{a}}{ }^{3}+0.0008 \mathrm{~K}_{\mathrm{a}}{ }^{2}-0.0004 \mathrm{~K}_{\mathrm{a}}+0.2203$ & 0.85632 & 0.84093 \\
4 & $\theta=-3 \mathrm{E}-05 \mathrm{~K}_{\mathrm{a}}{ }^{3}+0.0012 \mathrm{~K}_{\mathrm{a}}{ }^{2}-0.0082 \mathrm{~K}_{\mathrm{a}}+0.2577$ & 0.7794 & 0.75810 \\
5 & $\theta=-7 \mathrm{E}-05 \mathrm{~K}_{\mathrm{a}}{ }^{3}+0.0029 \mathrm{~K}_{\mathrm{a}}{ }^{2}-0.0279 \mathrm{~K}_{\mathrm{a}}+0.3231$ & 0.80565 & 0.78742 \\
6 & $\theta=-8 \mathrm{E}-05 \mathrm{~K}_{\mathrm{a}}{ }^{3}+0.0035 \mathrm{~K}_{\mathrm{a}}{ }^{2}-0.0378 \mathrm{~K}_{\mathrm{a}}+0.37$ & 0.84578 & 0.82983 \\
7 & $\theta=-2 \mathrm{E}-05 \mathrm{~K}_{\mathrm{a}}{ }^{3}+0.0006 \mathrm{~K}_{\mathrm{a}}{ }^{2}+0.0022 \mathrm{~K}_{\mathrm{a}}+0.2133$ & 0.68873 & 0.65760 \\
8 & $\theta=-2 \mathrm{E}-05 \mathrm{~K}_{\mathrm{a}}{ }^{3}+0.0002 \mathrm{~K}_{\mathrm{a}}{ }^{2}+0.0114 \mathrm{~K}_{\mathrm{a}}+0.1657$ & 0.78724 & 0.75823 \\
9 & $\theta=-2 \mathrm{E}-05 \mathrm{~K}_{\mathrm{a}}{ }^{3}+0.0007 \mathrm{~K}_{\mathrm{a}}{ }^{2}+0.0018 \mathrm{~K}_{\mathrm{a}}+0.2162$ & 0.84492 & 0.82991 \\
10 & $\theta=2 \mathrm{E}-05 \mathrm{~K}_{\mathrm{a}}{ }^{3}-0.0006 \mathrm{~K}_{\mathrm{a}}{ }^{2}+0.0155 \mathrm{~K}_{\mathrm{a}}+0.1734$ & 0.81869 & 0.80056 \\
11 & $\theta=-8 \mathrm{E}-05 \mathrm{~K}_{\mathrm{a}}{ }^{3}+0.0023 \mathrm{~K}_{\mathrm{a}}{ }^{2}-0.0124 \mathrm{~K}_{\mathrm{a}}+0.246$ & 0.84784 & 0.83262 \\
12 & $\theta=2 \mathrm{E}-05 \mathrm{~K}_{\mathrm{a}}{ }^{3}-0.0011 \mathrm{~K}_{\mathrm{a}}{ }^{2}+0.0255 \mathrm{~K}_{\mathrm{a}}+0.1223$ & 0.76353 & 0.73269 \\
13 & $\theta=-7 \mathrm{E}-05 \mathrm{~K}_{\mathrm{a}}{ }^{3}+0.002 \mathrm{~K}_{\mathrm{a}}{ }^{2}-0.0101 \mathrm{~K}_{\mathrm{a}}+0.2477$ & 0.83882 & 0.82270 \\
14 & $\theta=7 \mathrm{E}-05 \mathrm{~K}_{\mathrm{a}}{ }^{3}-0.0027 \mathrm{~K}_{\mathrm{a}}{ }^{2}+0.0417 \mathrm{~K}_{\mathrm{a}}+0.0615$ & 0.94273 & 0.93719 \\
15 & $\theta=-1 \mathrm{E}-05 \mathrm{~K}_{\mathrm{a}}{ }^{3}+0.0004 \mathrm{~K}_{\mathrm{a}}{ }^{2}+0.005 \mathrm{~K}_{\mathrm{a}}+0.2106$ & 0.85953 & 0.84594 \\
16 & $\theta=-2 \mathrm{E}-05 \mathrm{~K}_{\mathrm{a}}{ }^{3}+0.0006 \mathrm{~K}_{\mathrm{a}}{ }^{2}+0.0029 \mathrm{~K}_{\mathrm{a}}+0.181$ & 0.94149 & 0.93600 \\
17 & $\theta=2 \mathrm{E}-05 \mathrm{~K}_{\mathrm{a}}{ }^{3}-0.0008 \mathrm{~K}_{\mathrm{a}}{ }^{2}+0.0215 \mathrm{~K}_{\mathrm{a}}+0.1107$ & 0.86014 & 0.84661 \\
\hline
\end{tabular}


A Figura 3 traz os gráficos de dispersão dos resultados, os quais relacionam os valores de constante dielétrica aparente do solo com os teores de umidade volumétrica. Os gráficos mostram comportamento semelhante para as sondas, sendo o comportamento cúbico mais evidente na Figura $3 b$ do que na Figura 3a.

(a)

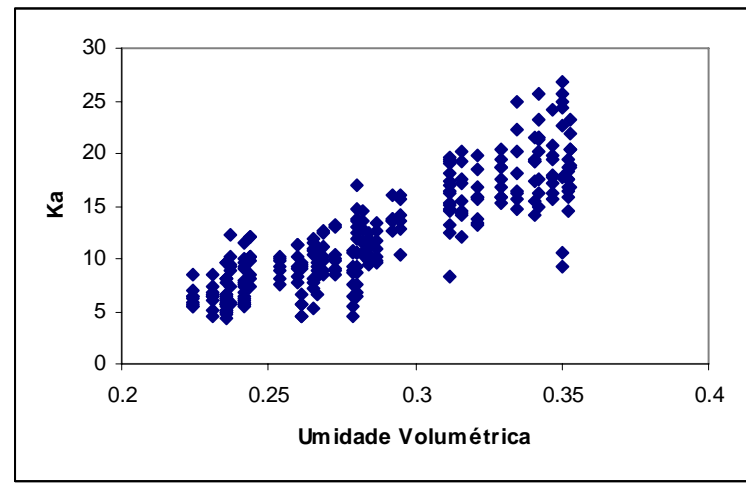

(b)

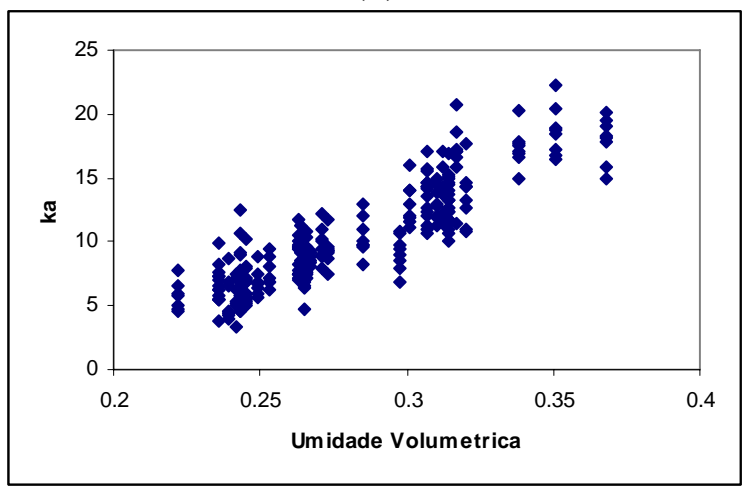

Figura 3. Gráficos de dispersão de $K_{\mathrm{a}}$ versus Umidade Volumétrica para: a) Sondas 1 a 8 e sonda 16; b) Sondas 9 a 15 e sonda 17.

\section{CONCLUSÕES}

1) $\mathrm{O}$ modelo que melhor se ajustou às dezessete sondas foi o modelo polinomial cúbico, comprovando os resultados obtidos por outros autores;

2) Os coeficientes das equações de calibração de todas as sondas mostraram-se semelhantes, indicando a viabilidade de emprego das sondas manufaturadas.

\section{AGRADECIMENTOS}

Aos Profs. Drs. Décio Lopes Cardoso e Miguel Angel Uribe Opazo, pela colaboração prestada;

À Prof ${ }^{a}$. Rosangela Villwock, pelo auxílio na confecção das sondas;

À Fundação Banco do Brasil, pelo apoio na aquisição do equipamento de TDR;

Ao Conselho Nacional de Desenvolvimento Científico e Tecnológico (CNPq) pela concessão da bolsa de mestrado a Dione Inês Christ Milani.

\section{REFERÊNCIAS}

COElHO, E. F.; VEllame, L. M.; FILHO, M. A. C.; LEDO, C. A. S. Desempenho de modelos de calibração de guias de onda acopladas a TDR e a multiplexadores em três tipos de solos. Revista Brasileira de Ciência do Solo, Viçosa, v. 30, n. 1, p. 23-30, 2006.

MANIERI, J. M.; VAZ, C. M. P.; MARIA, I. C. Sonda espiral de TDR para a medida da umidade no perfil do solo. Revista Brasileira de Ciência do Solo, Viçosa, v. 31, n. 2, p. 191-198, 2007.

NOBORIO, K. Measurement of soil water content and electrical conductivity by time domain reflectometry: a review. Computers and Electronics in Agriculture, Washington, v. 31, n. 3, p. 213-237, 2001. 
ROBINSON, D. A.; JONES S. B.; WRAITH, J. M.; OR, D.; FRIEDMAN, S. P. A Review of Advances in Dielectric and Electrical Conductivity Measurement in Soils Using Time Domain Reflectometry. Vadose Zone Journal, Madison, v. 2, n. 4, p. 444-475, 2003.

ROTH, C. H.; SCHULIN, R.; FLUHLER, H.; ATTINGER, W. Calibration of time-domain reflectometry for water content measurement using composite dielectric approach. Water Resources Research, v. 26, n. 10, p. 2267-2273, 1990.

SOILMOISTURE EQUIPMENT CORPORATION. Trase-Operating Instructions. Santa Barbara: S.E.C., 1998. 119 p.

TAVARES, M. H. F.; BUSATTA, O. J.; VILAS BOAS, M. A.; ORO, A. L. T. Desenvolvimento de aplicativo para um equipamento TDR. Irriga, Botucatu, v. 11, n. 4, p. 543-552, 2006.

TOMMASELLI, J. T. G.; BACCHI, O. O. S. Calibração de um equipamento de TDR para medida de umidade de solos. Pesquisa Agropecuária Brasileira, Brasília, v. 36, n. 9, p. 1145-1154, 2001.

TOPP, G. C.; DAVIS, J. L.; ANNAN, A. P. Electromagnetic determination of soil water content: measurements in coaxial transmission lines. Water Resources Research, Washington, v. 16, n. 3, p. 574-582, 1980.

VILLWOCK, R.; TAVARES, M. H. F.; VILAS BOAS, M. A. Calibração de um equipamento TDR em condições de campo. Irriga, Botucatu, v. 9, p. 82-88, 2004. 